\title{
On-surface mechanochemistry synthesis of non-alternant non-benzenoid aromatic compounds containing 4 to 8-membered rings
}

B. Mallada ${ }^{1,2 \dagger}$ B. de la Torre, ${ }^{1,2 \dagger *}$ J. I. Mendieta-Moreno, ${ }^{2}$ D. Nachtigallová1,3* A. Matěj, ${ }^{1,2}$ M. Matoušek, ${ }^{4}$ P. Mutombo, ${ }^{2}$ J. Brabec, ${ }^{4}$ L. Veis, ${ }^{4 *}$ T. Cadart,${ }^{5}$ M. Kotora, ${ }^{5 *}$ P. Jelínek,,${ }^{1,2 *}$

${ }^{1}$ Regional Centre of Advanced Technologies and Materials. Palacký University, 78371 Olomouc, Czech Republic.

${ }^{2}$ Institute of Physics, Czech Academy of Sciences, 16200 Prague, Czech Republic.

${ }^{3}$ Insitute of Organic Chemistry and Biochemistry, Czech Academy of Sciences, 16200 Prague, Czech Republic.

${ }^{4}$ J. Heyrovský Institute of Physical Chemistry, Czech Academy of Sciences, 18223 Prague, Czech Republic.

${ }^{5}$ Department of Organic Chemistry, Charles University, 16200 Prague, Czech Republic.

*Correspondence to: bruno.de@upol.cz (B.T.), dana.nachtigallova@uochb.cas.cz (D.N.), libor.veis@jh-inst.cas.cz (L.V.), martin.kotora@ natur.cuni.cz (M.K.), jelinekp@fzu.cz (P.J.)

$\dagger$ These authors contributed equally to this work.

\begin{abstract}
:
Synthesis of polycyclic aromatic hydrocarbons containing various non-benzenoid rings remains a big challenge facing contemporary organic chemistry despite a considerable effort made over the last decades. Herein, we present a novel route, employing on-surface mechanochemistry, to synthesize non-alternant polycyclic aromatic hydrocarbon containing up to four distinct kinds of non-benzenoid rings. We show that the surface-induced mechanical constrains imposed on strained helical reactants play a decisive role leading to the formation of products, energetically unfavorable in solution, with a peculiar ring current stabilizing the aromatic character of the $\pi$ conjugated system. Determination of the chemical and electronic structures of the most frequent product reveals its closed-shell character and low band gap. The present study renders a new route for the synthesis of novel non-alternant polycyclic aromatic hydrocarbons or other hydrocarbons not available by traditional approaches of organic chemistry in solution.
\end{abstract}

One Sentence Summary: We present a novel on-surface mechanochemistry route driven by surface confinement, which enables us to form new closed-shell non-alternant non-benzenoid aromatic compounds consisting of four to eight carbon rings not available by traditional organic synthesis.

\section{Main Text:}

35 The aim of organic chemistry is to search for novel reaction mechanisms and molecular compounds featuring interesting structural and material properties. Polycyclic aromatic hydrocarbons (PAHs) represent probably the most abundant and nowadays frequently studied organic molecules family. They have enormous industrial and environmental implications to everyday life, and, at the same time, they are assumed to play an essential role in the origin of Life 
(1). PAHs are composed of multiple carbon rings containing carbon and hydrogen atoms. The $\mathrm{sp}^{2}$ hybridization of $\mathrm{C}$ atoms and, consequently, a partial delocalization of $\pi$-electrons is responsible for the (anti)aromatic character of the rings. PAHs contain mostly benzene (aromatic) rings. However, other non-benzenoid rings, such as four, five, seven, eight, or even higher-membered rings, can be present in PAH structures, causing an internal charge transfer, giving rise to exciting material properties for opto- and nanoelectronics (2-4). However, these properties commensurate with their non-trivial electronic character, such as broken aromaticity or the introduction of a radical character, both deteriorating their stability and making their syntheses difficult. Indeed, only a very limited number of PAHs containing multiple non-benzenoid rings have been synthesized using traditional organic chemistry (5).

Recently introduced on-surface chemistry provides an interesting alternative for the syntheses of new chemical compounds inaccessible by traditional organic chemistry methods $(6,7)$. For example, unique and structurally diverse nanographene molecules have been synthesized on metal surfaces. Interestingly, these systems contain disordered regions of five- and seven-membered rings forming, e.g., the azulene regions, rather than homogenous hexagonal nanographene structures. Similarly, the on-surface syntheses have allowed producing curved nanographenes with regularly fused heptagons and pentagons (8), helical nanographenes with azulene units (9), nonbenzenoid nanographenes (10), or polyazulene nanoribbons (11). It is fair to admit that such rearrangements have also been occasionally observed in solution chemistry during the Scholl reaction $(12,13)$.

The stability of molecules is often determined by a strain in their molecular framework caused by steric repulsions, usually being the primary cause of their molecular structure rearrangements (and other desirable or undesirable processes) to a variety of products. However, such rearrangements are often difficult to induce. The adsorption of the strained molecules on metal surfaces can promote such processes much easier and induce hitherto otherwise unlikely reaction pathways (14, 15). The feasibility of such processes results, by no doubt, from multiple overlaps between the orbitals of the adsorbed molecule and the metal surface $(6,7)$. One of such well-documented processes in nanographene molecules is Stone-Wales rearrangement $(16,17)$.

One of the potentially exciting pathways for the preparation of nanographene molecules that possess disordered regions is the surface transformation of compounds having rigid and strained 3D-architectures. In this respect, helicenes and other types of helical molecules represent attractive candidates for studying strain-induced reactions on metal surfaces and their eventual planarization being the ultimate target. The planarization is usually accompanied by bond reorganizations (skeletal rearrangements) in their molecular scaffold to accommodate the final planar molecular arrangement optimizing dispersive interaction with the underlying substrate. Thus, the interaction between the molecule's framework and the metal surface often induced otherwise tricky or even not accessible processes to occur either in solution or in the gas-phase. The conversion of a [7] helicene to planar coranulene derivative on $\operatorname{Ag}(111)$ surface (18) or the transformation of helical compounds with azuleno moieties to planar species with fulvaleno segments on $\mathrm{Cu}(001)$ surface (19) demonstrates such surface-induced reactions. Although several reports on the deposition of helical compounds on metal surfaces have been published (20-22), their further transformations 
have not been studied yet in detail and, thus, the above-mentioned studies $(18,19)$ represent unique reports on the planarization of helical compounds in this potentially attractive but hitherto unexplored area.

Recently we have developed a new approach to synthesize series of structurally rigid [7]helical indenofluorenes (7-IFs), (23) systems with curved architecture, which are potentially attractive substrates for different structural modifications, e.g., strain-induced rearrangement, cyclodehydrogenation, and planarization. For this purpose, we have synthesized an indenofluorene based system (see Fig. 1A and SOM) and explored the possibilities of its transformation via cyclodehydrogenation to form unusual planar aromatic species.

We deposited the precursor via thermal sublimation at $450 \mathrm{~K}$ from a Knudsen cell onto an $\mathrm{Au}(111)$ surface held at room temperature under (ultra-high vacuum) UHV conditions. The freshly prepared sample was inspected by cryogenic STM/nc-AFM (scanning tunneling microscopy/non-contact Atomic force microscopy) at 4.2 Kelvin. Figure S1 displays a representative set of STM images showing the evaluation of molecules on the surface upon gradual annealing ranging from 425 to $625 \mathrm{~K}$. At $425 \mathrm{~K}$, the precursors remain stable and arrange in two-dimensional islands with no apparent long-range order. Subsequent thermal annealing of the molecule-decorated Au surface to $\mathrm{T} \sim 525 \mathrm{~K}$ for $30 \mathrm{~min}$ gives rise to the thermally induced chemical transformation of the precursor into significantly different molecular products.

Figure 1B shows a characteristic STM image of the surface after the annealing procedure at T $575 \mathrm{~K}$ revealing the precursor's chemical transformation, including its planarization. Planar structures of the products are unambiguously distinguishable in STM images by lack of bright spots in their images. Four observed planar objects detected in the STM image are labeled 1, 2, 3, and 4. Their chemical structures were determined using high-resolution nc-AFM measurements with a CO-functionalized tip $(24,25)$. Figure $1 \mathrm{C}$ shows constant height nc-AFM images of all the planar products P1, P2, P3, P4, and P5 observed after annealing the sample at T $\geq 525 \mathrm{~K}$. Total energy DFT simulations (26) of possible products formed on $\mathrm{Au}(111)$ surface predict their planarity and positioning at $\sim 3.3 \AA$ above the surface (Fig. S2), resulting from the attractive dispersion interactions between $\mathrm{P}(1-4)$ molecules and the surface. Very good agreement between experimental and simulated (27) high-resolution nc-AFM images (compare Fig. 1C and d) allows us to unambiguously assign the structure of the planar products, which contain four-to-eightmembered rings (see Fig. 1E). Further annealing at an elevated temperature up to $625 \mathrm{~K}$ does not alter the occurrence of the planar structures but enhances the frequency of oligomers and other unidentifiable objects, as shown in Fig. S1. At $575 \mathrm{~K}$, the planar structures represent $~ 30 \%$ of the total molecular structures, while most products comprise other minority non-planar monomers and fused oligomers (Fig. 1B).

Statistical analyses performed on several samples, including hundreds of planar products, exhibit the relative incidences of products $\mathrm{P} 1: \mathrm{P} 2: \mathrm{P} 3: \mathrm{P} 4: \mathrm{P} 5$ as 32:25:22:15:6. The most populated $\mathrm{P} 1$ has 
the most exotic chemical structure consisting of four to eight-membered rings and thus, will be the main focus of our investigations reported in the rest of the manuscript.

Figure S2 displays fully optimized structures of P1-P5 products on $\mathrm{Au}(111)$ surface performed at the DFT level. From the chemical structure of the precursor and the products, we proposed a tentative course of reaction steps (see Scheme S1 and related discussion). P2 and P3 chemical structures have one carbon atom less than the precursor, suggesting a demethylation process upon their formation (28). On the other hand, P1, P4, and P5 products conserve the number of carbon atoms during the reaction course. Thus, the product formations proceed via subsequent dehydrogenation processes, removing the same number of hydrogens in all three cases. The same chemical content of P1, P4, and P5 allows for a direct estimate of their relative stabilities, comparing their total energies. Interestingly, the total energy DFT calculations predict larger thermodynamical stabilities of P4 and P5 (by 2.2 and $0.9 \mathrm{eV}$, respectively) than P1 (see Fig. 1C), in strike contrast with their relative abundances observed in the experiment. To understand these discrepancies, possible reaction pathways towards P1 and P4 formations will be analyzed below. A detailed analyses of reaction paths leading to P2 and P3 are beyond the scope of this paper and will be discussed in detail elsewhere (a tentative scheme is proposed in Scheme S1).

Deposition of the highly non-planar helical precursor on the surface (see Fig S2) causes internal stress imposed on the molecule resulting from the competition between attractive dispersion interactions and steric repulsions between non-planar phenyl groups, and/or helical part of precursor and the underlying metallic substrate. DFT simulations predict that such competition leads to an asymmetric arrangement of phenyl groups with methyl groups pointing out of the surface (Fig. S2). This asymmetric arrangement is determined by the chirality of the molecule's helical part (see Fig 2Aa). Importantly, the surface-induced geometrical constrain determines the course of reactions, as will be demonstrated below.

Possible reaction pathways from the precursor towards $\mathrm{P} 1$ and $\mathrm{P} 4$ products are displayed in Fig. S3 and summarized in Fig. 2A. The reaction schemes include dehydrogenation accompanied by annulation or cyclization processes divided into four consecutive steps.

Two possible reaction channels starting from the precursor are considered in the first step: (i) construction of six-membered ring via annulation of a methyl group with the central carbon of fluorene unit accompanied by dehydrogenation processes, leading to intermediate A1, shown in Figures $2 \mathrm{~A}$ and S3 and (ii) dehydrogenation/annulation process with fusing the helical part and cyclobutadiene formation (see intermediate A2 in Fig. S3). The simulations, however, predict free energy of A2 intermediate being by about $1 \mathrm{eV}$ lower than A1. Thus, the first reaction channel is considered to be more favorable and discussed below.

The course of the initial step of the reaction, in particular, what benzene ring will first planarize during the dehydrogenation of methyl groups (29), is directed by their asymmetric position imposed by the underlying surface; i.e., the distances of the bonds, labeled as $\mathrm{d} 1$ and $\mathrm{d} 2$ (see Fig. 
S4), to be formed between the carbon atoms of methyl groups and matching central $\mathrm{C}$ atoms of fluorene. The relevant analysis includes the determination of the kinetic control over the reaction, i.e., the calculation of the activation energies for the two products. Such an approach, however, requires considering multiple dehydrogenations, which greatly complicates and questions the reliability of such calculations. Alternatively, probabilities of these steps can be determined using an assumption that the reaction rates depend on the reaction coordinates' behavior around the equilibrium position of the initial state. In other words, the rates of the two reaction pathways can be qualitatively compared based on the time-course distribution of $\mathrm{d} 1$ and $\mathrm{d} 2$ distances along the reaction coordinates at a given temperature. For this purpose, molecular dynamics (MD) simulations, employing the hybrid quantum mechanics/molecular mechanics (QM/MM) methods (30-32) were performed to obtain temporal evolution of $\mathrm{d} 1$ and $\mathrm{d} 2$ reaction coordinates (Fig. S4) and the probability distribution for the given intermediate (Fig. S4). In these simulations, the precursor and intermediates are treated at the QM level, while the metallic surface is described at the MM level. The histogram of $\mathrm{d} 1$ and $\mathrm{d} 2$ distances (Fig S4) shows a significantly shorter timeaverage distance between methyl carbon and the central fluorene carbon located on the helical part of the molecule closer to the metallic surface (d1 in Fig S4). The resulting reaction channel thus leads to A1 (Figs 2A, S3, and S4), i.e., the formation of the six-membered ring on the side of the molecule relevant to the shorter $\mathrm{d} 1$ distance and, consequently, planarization of the appropriate part of the molecule. The planarization stabilizes the system by additional dispersion attractive forces due to a newly formed ring with $\pi$-electrons interacting with the substrate. It is noteworthy that this reaction step determines the resulting chirality of the P1.

The decisive step determining the formation of $\mathrm{P} 1$ or $\mathrm{P} 4$ product represents the dehydrogenation/annulation process with planarization of the second phenyl ring, which involves the methyl group. This bifurcation point occurs either prior to or after the dehydrogenation/annulation process with fusing the helical part, i.e., at intermediates A1 or B3 (see Fig S3). The two possible channels, related to the seven- or six-membered rings' construction, describe the phenyl ring planarization. Similar to the first step, the surface-imposed asymmetrical arrangement of the methyl group in A1 or B3 to adjacent benzene or fluorene determines the probabilities of the two channels and the course of the reaction. From A1, these channels lead to either seven-membered (B1 intermediate) or six-membered (B2 intermediate) ring construction, and, consequently, the probabilities of $\mathrm{A} 1 \rightarrow \mathrm{B} 1$ and $\mathrm{A} 1 \rightarrow \mathrm{B} 2$ processes determine the relative yield of P1 and P4, as shown in Fig. 2.

The MD simulations (see Figures 2D and S5 for the results of the distance-time distributions) predict B1 more likely than B2; thus, P1 is preferred over P4. From B3, the alternative channels for $\mathrm{P} 1$ and $\mathrm{P} 4$ formation are $\mathrm{B} 3 \rightarrow \mathrm{C} 2 \rightarrow \mathrm{P} 1$ and $\mathrm{B} 3 \rightarrow \mathrm{P} 4$, respectively. Like in the previous case, MD simulations predict the formation of the seven-membered ring (Fig. S6), i.e., following the former reaction path, which is more feasible due to the shorter $\mathrm{d} 1$ reaction coordinate. These observations rationalize the larger abundance of $\mathrm{P} 1$ over the more stable $\mathrm{P} 4$. As for the formation 40 of P5, it can be formed from P4 by a simple 1,2-shift or from P1 by a series of 1,2-shifts (Scheme $\mathrm{S} 1)$.

Fig. 3A presents constant height dI/dV spectra acquired over two different positions of the P1 shown on the inset. The $\mathrm{dI} / \mathrm{dV}$ spectra reveals three distinct peaks at $-700,-400$, and $300 \mathrm{meV}$ 
respectively assigned to HOMO-1, HOMO, and LUMO (HOMO and LUMO stand for the highest occupied and lowest unoccupied molecular orbitals, respectively) and providing molecular band gap of $0.7 \mathrm{eV}$. The calculated DFT-PBE0 band gap of $\sim 1.4 \mathrm{eV}$ of free-standing P1 product concurs well with the experimental observation. We expect that in the presence of the underlying metallic surface, these values reduce (33). The experimental $\mathrm{dI} / \mathrm{dV}$ spectra are lack of any zero-bias peak or inelastic electron tunneling signal, which indicates the closed-shell ground state of P1. Its closed-shell character is also confirmed by DFT-PBE0 and DMRG calculations of the freestanding molecule. The DMRG (34) calculations were performed with the active space constructed from a full $\pi$-orbital space ( 42 electrons in 42 orbitals), resulting in the occupancies of the highest occupied and lowest unoccupied natural orbitals of 1.80 and 0.23 , respectively.

Figure 3B shows the constant current $\mathrm{dI} / \mathrm{dV}$ maps at the corresponding energies resolving spatial localization of electron density of the frontier orbitals. Namely, dI/dV maps of LUMO reveal strong spatial localization in the vicinity of the seven-membered ring. This picture is fully supported by the DFT-calculated dI/dV map (35) of canonical LUMO, showing the same localization pattern, see Fig 3C. The remaining calculated maps of occupied frontier orbitals show fairly good agreement with their experimental counterparts as well, thus confirming the orbital assignation.

High-resolution nc-AFM imaging allows direct visualization of the chemical structure of the P1 product revealing the presence of all four- to eight-member rings, see Fig. 4A. Namely, the presence of the seven-membered ring can be unambiguously determined from a careful analysis of bond lengths in the nc-AFM images of the P1 and P4 products (Fig. S7). The presence of multiple non-benzenoid rings causes the localization of molecular orbitals at distinct parts of P1 introducing an internal charge transfer within the molecule (Fig. 4C). Thus, the submolecular contrast seen in the high-resolution nc-AFM images is heavily affected by the electrostatic interaction between CO-tip and P1 (36), altering the apparent $\mathrm{C}-\mathrm{C}$ bond lengths with respect to real ones and making the bond-order analysis (37) from the nc-AFM images ambiguous. Despite a good overall agreement between experimental and calculated nc-AFM images (see Fig 4A, 4B and Fig. S8), the comparison of the experimental and calculated apparent bond lengths is not fully conclusive.

Thus, to assign the most representative resonant structure of $\mathrm{P} 1$, we adopted an alternative approach employing the mutual information of atomic-like orbitals that form $\pi$ bonds (see SOM). Mutual information of individual bonds (Fig. 4D) was obtained from DMRG calculations. The resulting structure (Fig. 4F) contains three disjoint Clar's sextets, i.e., benzoid moieties (38). Alternative resonant structures consisting of more such sextets (five being the maximum number, see Fig. S9) lead to the biradical character of the structure increasing its instability.

Next, we address the aromatic character of P1. For systems consisted of several fused rings, the aromaticity criteria can be applied either to the whole system, i.e., counting all $\pi$-electrons in the systems, or considering only $\pi$-electrons on the periphery, thus distinguishing between the global and peripheral aromaticity (see e.g., (39)). Application of the most popular Hückel's (40-42) and 
Baird's rules (43) on P1 in the closed-shell ground state configuration provides contradictory results: the global aromaticity $(42$, i.e. $(4 n+2) \pi$-electrons) and peripheral antiaromaticity (28, i.e. (4n) $\pi$-electrons). Despite the frequent use of these rules, generalized by Soncini and Fowler (44), they hold strictly for monocyclic systems and can be violated in polycyclic systems.

In the following, we report on the analyses performed using various methods, which in combination can resolve the aromaticity character of P1. Tab S1 summarizes results based on the local aromaticities of individual rings obtained with the following approaches: the generalized harmonic oscillator model (HOMA) $(45,46)$ using the mutual information of atomic-like orbitals forming $\pi$ bonds from DMRG (see Table S1), the nucleus-independent chemical shifts (NICS) (47), the isotropic magnetic shielding (IMS) (48) (see Fig. S10). NICS and IMS methods agree on strong antiaromaticity of the four-membered ring and weak antiaromaticities of the seven- and eight-membered rings, respectively, as well as one aromatic (5-II) and two antiaromatic (5-I, 5III) five-membered rings. Agreement of NICS and ISM results is also reached for aromaticity indexes of the six-membered rings. These values are, however, in contrast with the HOMA results. In particular, NICS and ISM methods predict the strong aromaticity of the inner ring (6-IV, see Fig S10 and Table S1), while its aromaticity is very low according to the HOMA-DMRG calculations. Interestingly, the results of NICS and ISM calculations, i.e., the strong aromaticity and Clar's sextet in 6-IV, contradict the existence of the closed-shell resonant structure, hence the experimental, as well as theoretical DFT and DMRG results.

The local aromaticity analyses described above do not allow for a definite assignment of the whole system's aromatic character. The concept of conjugated circuits $(49,50)$, applied on the DMRG resonance structure (Fig. 4D), predicts that $\mathrm{P} 1$ is partially aromatic, with the aromaticity degree of 95\% (see Fig S12 for details).

Probably the most complex picture of the aromaticity character of $\mathrm{P} 1$ is obtained by calculations of the anisotropy of the induced current density (ACID) (51). The ACID map (Fig. 4F) reveals the interactions of ring currents of each individual ring, enhancing or suppressing on the boundary with neighboring rings, depending on their mutual orientations. Indeed, the strong negative (aromatic) NICS and ISM values on the inner 6-IV ring are caused by the presence of synergic anti-aromatic currents on two adjacent 5-rings, see Fig. 4F.

Similarly, IMS ad NICS values predict the weak antiaromaticity of the 8-ring in contrast to its weak aromatic character predicted with ACID. The latter result originates from the current enhancement due to similarly oriented current on joint edges with 6-IV and 4- rings, and weakening by the opposite current directions on the neighbor 5- and 6-rings, see Fig. S13.

The ACID map also shows the clockwise direction of the main current ring on the P1 periphery revealing its aromatic character. Although in disagreement with $4 n+2$ rule of aromaticity, all $\pi$ electrons (28) are involved in this current. The aromaticity and, thus, the stabilization in the closedshell electronic structure are maintained by avoiding peripheral bonds of antiaromatic 4, 5-I and 
5-III rings. This indicates that the closed-shell ground state character in non-alternant nonbenzenoid aromatic compounds can be reached via the development of a complex peripheral ring current excluding certain molecular parts and suppressing the local aromatic/antiaromatic character of rings.

We anticipate that the mechano-chemical approach employing internal stress of non-planar molecules on surfaces might enable the formation of new non-benzenoid PAH compounds, not available via traditional synthetic methods in solution or on surfaces. Such non-benzenoid PAH nanostructures offer a rich playground to reach interesting optical and magnetic properties of carbon-based nanostructures with closed-shell or radical character. Thus, we believe that this novel synthetic route is not only just a synthetic curiosity but it can stimulate further research effort to form non-benzenoid PAH complexes, including polymers, which can open new routes to design novel spintronics and optoelectronics devices.

\section{References}

1. P. Ehrenfreund, S. Rasmussen, J. Cleaves, L. Chen, Experimentally tracing the key steps in the origin of life: The aromatic world. Astrobiology. 6, 490-520 (2006).

2. J. E. Anthony, Functionalized Acenes and Heteroacenes for Organic Electronics. Chem. Rev. 106, 5028-5048 (2006).

3. J. E. Anthony, The larger acenes: Versatile organic semiconductors. Angew. Chemie - Int. Ed. 47, 452-483 (2008).

4. Y. Wang, B. Liu, C. W. Koh, X. Zhou, H. Sun, J. Yu, K. Yang, H. Wang, Q. Liao, H. Y. Woo, X. Guo, Facile Synthesis of Polycyclic Aromatic Hydrocarbon (PAH)-Based Acceptors with Fine-Tuned Optoelectronic Properties: Toward Efficient Additive-Free Nonfullerene Organic Solar Cells. Adv. Energy Mater. 9, 1803976 (2019).

5. X. S. Zhang, Y. Y. Huang, J. Zhang, W. Meng, Q. Peng, R. Kong, Z. Xiao, J. Liu, M. Huang, Y. Yi, L. Chen, Q. Fan, G. Lin, Z. Liu, G. Zhang, L. Jiang, D. Zhang, Dicyclohepta[ijkl,uvwx]rubicene with Two Pentagons and Two Heptagons as a Stable and Planar Non-benzenoid Nanographene. Angew. Chemie - Int. Ed. 59, 3529-3533 (2020).

6. S. Clair, D. G. De Oteyza, Controlling a Chemical Coupling Reaction on a Surface: Tools and Strategies for On-Surface Synthesis. Chem. Rev. 119, 4717-4776 (2019).

7. T. Wang, J. Zhu, Confined on-surface organic synthesis: Strategies and mechanisms. Surf. Sci. Rep. 74 (2019), pp. 97-140.

35 8. I. C. Y. Hou, Q. Sun, K. Eimre, M. Di Giovannantonio, J. I. Urgel, P. Ruffieux, A. Narita, R. Fasel, K. Müllen, On-Surface Synthesis of Unsaturated Carbon Nanostructures with Regularly Fused Pentagon-Heptagon Pairs. J. Am. Chem. Soc. 142, 10291-10296 (2020).

9. N. Ogawa, Y. Yamaoka, H. Takikawa, K. I. Yamada, K. Takasu, Helical Nanographenes Embedded with Contiguous Azulene Units. J. Am. Chem. Soc. 142, 13322-13327 (2020).

40 10. T. G. Lohr, J. I. Urgel, K. Eimre, J. Liu, M. Di Giovannantonio, S. Mishra, R. Berger, P. Ruffieux, C. A. Pignedoli, R. Fasel, X. Feng, On-Surface Synthesis of Non-Benzenoid Nanographenes by Oxidative Ring-Closure and Ring-Rearrangement Reactions. J. Am. Chem. Soc. (2020).

11. Q. Fan, D. Martin-Jimenez, D. Ebeling, C. K. Krug, L. Brechmann, C. Kohlmeyer, G. Hilt, W. Hieringer, A. Schirmeisen, J. M. Gottfried, Nanoribbons with Nonalternant Topology from Fusion of Polyazulene: Carbon Allotropes beyond Graphene. J. Am. Chem. Soc. 141, 
17713-17720 (2019).

12. S. Nobusue, K. Fujita, Y. Tobe, Skeletal Rearrangement of Twisted Polycyclic Aromatic Hydrocarbons under Scholl Reaction Conditions. Org. Lett. 19, 3227-3230 (2017).

13. Y. Han, Z. Xue, G. Li, Y. Gu, Y. Ni, S. Dong, C. Chi, Formation of Azulene-Embedded Nanographene: Naphthalene to Azulene Rearrangement During the Scholl Reaction. Angew. Chemie - Int. Ed. 59, 9026-9031 (2020).

14. J. Su, X. Wu, S. Song, M. Telychko, J. Lu, Substrate induced strain for on-surface transformation and synthesis. Nanoscale. 12, 7500-7508 (2020).

15. M. Telychko, J. Su, A. Gallardo, Y. Gu, J. I. Mendieta-Moreno, D. Qi, A. Tadich, S. Song, P. Lyu, Z. Qiu, H. Fang, M. J. Koh, J. Wu, P. Jelínek, J. Lu, Strain-Induced Isomerization in One-Dimensional Metal-Organic Chains. Angew. Chemie - Int. Ed. 58, 18591-18597 (2019).

16. A. J. Stone, D. J. Wales, Theoretical studies of icosahedral C60 and some related species. Chem. Phys. Lett. 128, 501-503 (1986).

17. E. Brayfindley, E. E. Irace, C. Castro, W. L. Karney, Stone-Wales Rearrangements in Polycyclic Aromatic Hydrocarbons: A Computational Study. J. Org. Chem. 80, 3825-3831 (2015).

18. O. Stetsovych, M. Švec, J. Vacek, J. V. Chocholoušová, A. Janík, J. Rybáek, K. Kosmider, I. G. Stará, P. Jelínek, I. Starý, From helical to planar chirality by on-surface chemistry. Nat. Chem. 9, 213-218 (2017).

19. A. Shiotari, T. Nakae, K. Iwata, S. Mori, T. Okujima, H. Uno, H. Sakaguchi, Y. Sugimoto, Strain-induced skeletal rearrangement of a polycyclic aromatic hydrocarbon on a copper surface. Nat. Commun. 8, 16089 (2017).

20. A. Shiotari, K. Tanaka, T. Nakae, S. Mori, T. Okujima, H. Uno, H. Sakaguchi, Y. Sugimoto, Chiral Discrimination and Manipulation of Individual Heptahelicene Molecules on $\mathrm{Cu}(001)$ by Noncontact Atomic Force Microscopy. J. Phys. Chem. C. 122, 4997-5003 (2018).

21. H. Zhang, H. Liu, C. Shen, F. Gan, X. Su, H. Qiu, B. Yang, P. Yu, Chiral recognition of hexahelicene on a surface via the forming of asymmetric heterochiral trimers. Int. J. Mol. Sci. 20 (2019).

22. A. Mairena, J. I. Mendieta, O. Stetsovych, A. Terfort, I. G. Stará, I. Starý, P. Jelínek, K. H. Ernst, Heterochiral recognition among functionalized heptahelicenes on noble metal surfaces. Chem. Commun. 55, 10595-10598 (2019).

23. R. P. Kaiser, D. Nečas, T. Cadart, R. Gyepes, I. Císařová, J. Mosinger, L. Pospíšil, M. Kotora, Straightforward Synthesis and Properties of Highly Fluorescent [5]- and [7]-Helical Dispiroindeno[2,1-c]fluorenes. Angew. Chemie - Int. Ed. 58, 17169-17174 (2019).

24. L. Gross, F. Mohn, N. Moll, P. Liljeroth, G. Meyer, The chemical structure of a molecule resolved by atomic force microscopy. Science 325, 1110-1114 (2009).

25. P. Jelinek, High resolution SPM imaging of organic molecules with functionalized tips. $J$. Phys. Condens. Matter. 29, 343002 (2017).

26. V. Blum, R. Gehrke, F. Hanke, P. Havu, V. Havu, X. Ren, K. Reuter, M. Scheffler, Ab initio molecular simulations with numeric atom-centered orbitals. Comput. Phys. Commun. 180, 2175-2196 (2009).

27. P. Hapala, G. Kichin, C. Wagner, F. S. Tautz, R. Temirov, P. Jelínek, Mechanism of highresolution STM/AFM imaging with functionalized tips. Phys. Rev. B - Condens. Matter Mater. Phys. 90 (2014).

28. F. Eisenhut, T. Lehmann, A. Viertel, D. Skidin, J. Krüger, S. Nikipar, D. A. Ryndyk, C. 
Joachim, S. Hecht, F. Moresco, G. Cuniberti, On-Surface Annulation Reaction Cascade for the Selective Synthesis of Diindenopyrene. ACS Nano. 11, 12419-12425 (2017).

29. J. Su, M. Telychko, P. Hu, G. Macam, P. Mutombo, H. Zhang, Y. Bao, F. Cheng, Z. Q. Huang, Z. Qiu, S. J. R. Tan, H. Lin, P. Jelínek, F. C. Chuang, J. Wu, J. Lu, Atomically precise bottom-up synthesis of -extended [5]triangulene. Sci. Adv. 5 (2019).

30. J. I. Mendieta-Moreno, R. C. Walker, J. P. Lewis, P. Gómez-Puertas, J. Mendieta, J. Ortega, Fireball / amber: An efficient local-orbital DFT QM/MM method for biomolecular systems. J. Chem. Theory Comput. 10, 2185-2193 (2014).

31. H. Heinz, T. J. Lin, R. Kishore Mishra, F. S. Emami, Thermodynamically consistent force fields for the assembly of inorganic, organic, and biological nanostructures: The INTERFACE force field. Langmuir. 29, 1754-1765 (2013).

32. J. P. Lewis, P. Jelínek, J. Ortega, A. A. Demkov, D. G. Trabada, B. Haycock, H. Wang, G. Adams, J. K. Tomfohr, E. Abad, H. Wang, D. A. Drabold, Advances and applications in the FIREBALL ab initio tight-binding molecular-dynamics formalism. Phys. Status Solidi Basic Res. 248, 1989-2007 (2011).

33. J. B. Neaton, M. S. Hybertsen, S. G. Louie, Renormalization of molecular electronic levels at metal-molecule interfaces. Phys. Rev. Lett. 97 (2006).

34. G. K. L. Chan, S. Sharma, The density matrix renormalization group in quantum chemistry. Annu. Rev. Phys. Chem. 62, 465-481 (2011).

35. O. Krejčí, P. Hapala, M. Ondráček, P. Jelínek, Principles and simulations of high-resolution STM imaging with a flexible tip apex. Phys. Rev. B. 95 (2017).

36. P. Hapala, M. Švec, O. Stetsovych, N. J. Van Der Heijden, M. Ondráčk, J. Van Der Lit, P. Mutombo, I. Swart, P. Jelínek, Mapping the electrostatic force field of single molecules from high-resolution scanning probe images. Nat. Commun. 7, 11560 (2016).

37. L. Gross, F. Mohn, N. Moll, B. Schuler, A. Criado, E. Guitián, D. Peña, A. Gourdon, G. Meyer, Bond-order discrimination by atomic force microscopy. Science 337, 1326-1329 (2012).

38. E. Clar, The aromatic sextet. (Wiley-Interscience, London, 1972).

39. H. Miyoshi, S. Nobusue, A. Shimizu, Y. Tobe, Non-alternant non-benzenoid kekulenes: The birth of a new kekulene family. Chem. Soc. Rev. 44 (2015), pp. 6560-6577.

40. E. Hückel, Quantentheoretische Beiträge zum Benzolproblem - I. Die Elektronenkonfiguration des Benzols und verwandter Verbindungen. Zeitschrift für Phys. 70, 204-286 (1931).

41. E. Hückel, Quanstentheoretische Beiträge zum Benzolproblem - II. Quantentheorie der induzierten Polaritäten. Zeitschrift für Phys. 72, 310-337 (1931).

42. E. Hückel, Quantentheoretische Beiträge zum Problem der aromatischen und ungesättigten Verbindungen. III. Zeitschrift für Phys. 76, 628-648 (1932).

43. N. C. Baird, Quantum Organic Photochemistry. II. Resonance and Aromaticity in the Lowest $3 \pi \pi *$ State of Cyclic Hydrocarbons. J. Am. Chem. Soc. 94, 4941-4948 (1972).

44. A. Soncini, P. W. Fowler, Ring-current aromaticity in open-shell systems. Chem. Phys. Lett. 450, 431-436 (2008).

45. J. Kruszewski, T. M. Krygowski, Definition of aromaticity basing on the harmonic oscillator model. Tetrahedron Lett. 13, 3839-3842 (1972).

46. S. Ostrowski, J. C. Dobrowolski, What does the HOMA index really measure? RSC Adv. 4, 44158-44161 (2014).

47. Z. Chen, C. S. Wannere, C. Corminboeuf, R. Puchta, P. von Ragué Schleyer, Nucleus- 
independent chemical shifts (NICS) as an aromaticity criterion. Chem. Rev. 105 (2005), pp. 3842-3888.

48. B. J. Lampkin, P. B. Karadakov, B. VanVeller, Detailed Visualization of Aromaticity Using Isotropic Magnetic Shielding. Angew. Chemie - Int. Ed. 59, 19275-19281 (2020).

49. M. Randić, Conjugated circuits and resonance energies of benzenoid hydrocarbons. Chem. Phys. Lett. 38, 68-70 (1976).

50. M. Randicć, Aromaticity and Conjugation. J. Am. Chem. Soc. 99, 444-450 (1977).

51. D. Geuenich, K. Hess, F. Köhler, R. Herges, Anisotropy of the induced current density (ACID), a general method to quantify and visualize electronic delocalization. Chem. Rev. (2005).

52. I. Horcas, R. Fernández, J. M. Gómez-Rodríguez, J. Colchero, J. Gómez-Herrero, A. M. Baro, WSXM: A software for scanning probe microscopy and a tool for nanotechnology. Rev. Sci. Instrum. 78, 013705 (2007).

53. J. P. Perdew, K. Burke, M. Ernzerhof, Generalized gradient approximation made simple. Phys. Rev. Lett. 77, 3865-3868 (1996).

54. A. Tkatchenko, M. Scheffler, Accurate molecular van der Waals interactions from groundstate electron density and free-atom reference data. Phys. Rev. Lett. 102, 073005 (2009).

55. P. V. R. Schleyer, C. Maerker, A. Dransfeld, H. Jiao, N. J. R. Van Eikema Hommes, Nucleus-independent chemical shifts: A simple and efficient aromaticity probe. J. Am. Chem. Soc. 118, 6317-6318 (1996).

56. R. Herges, D. Geuenich, Delocalization of electrons in molecules. J. Phys. Chem. A. 105, 3214-3220 (2001).

57. M. J. Frisch, G. W. Trucks, H. B. Schlegel, G. E. Scuseria, M. A. Robb, J. R. Cheeseman, G. Scalmani, V. Barone, B. Mennucci, G. A. Petersson, H. Nakatsuji, M. Caricato, X. Li, H. P. Hratchian, A. F. Izmaylov, J. Bloino, G. Zheng, J. L. Sonnenberg, M. Hada, M. Ehara, K. Toyota, R. Fukuda, J. Hasegawa, M. Ishida, T. Nakajima, Y. Honda, O. Kitao, H. Nakai, T. Vreven, J. A. Montgomery, J. E. Peralta, F. Ogliaro, M. Bearpark, J. J. Heyd, E. Brothers, K. N. Kudin, V. N. Staroverov, R. Kobayashi, J. Normand, K. Raghavachari, A. Rendell, J. C. Burant, S. S. Iyengar, J. Tomasi, M. Cossi, N. Rega, J. M. Millam, M. Klene, J. E. Knox, J. B. Cross, V. Bakken, C. Adamo, J. Jaramillo, R. Gomperts, R. E. Stratmann, O. Yazyev, A. J. Austin, R. Cammi, C. Pomelli, J. W. Ochterski, R. L. Martin, K. Morokuma, V. G. Zakrzewski, G. A. Voth, P. Salvador, J. J. Dannenberg, S. Dapprich, A. D. Daniels, Farkas, J. B. Foresman, J. V Ortiz, J. Cioslowski, D. J. Fox, Gaussian 09, Revision B.01. Gaussian 09, Revis. B.01, Gaussian, Inc., Wallingford CT (2009).

58. A. D. Becke, Density-functional thermochemistry. III. The role of exact exchange. J. Chem. Phys. 98, 5648-5652 (1993).

59. C. Lee, W. Yang, R. G. Parr, Development of the Colle-Salvetti correlation-energy formula into a functional of the electron density. Phys. Rev. B. 37, 785-789 (1988).

60. S. H. Vosko, L. Wilk, M. Nusair, Accurate spin-dependent electron liquid correlation energies for local spin density calculations: a critical analysis. Can. J. Phys. 58, 1200-1211 (1980).

61. T. H. Dunning, Gaussian basis sets for use in correlated molecular calculations. I. The atoms boron through neon and hydrogen. J. Chem. Phys. 90, 1007-1023 (1989).

62. A. D. Becke, Density-functional exchange-energy approximation with correct asymptotic behavior. Phys. Rev. A. 38, 3098-3100 (1988).

63. S. Grimme, S. Ehrlich, L. Goerigk, Effect of the damping function in dispersion corrected 
density functional theory. J. Comput. Chem. 32, 1456-1465 (2011).

64. M. A. Basanta, Y. J. Dappe, P. Jelínek, J. Ortega, Optimized atomic-like orbitals for firstprinciples tight-binding molecular dynamics. Comput. Mater. Sci. 39, 759-766 (2007).

65. S. Szalay, M. Pfeffer, V. Murg, G. Barcza, F. Verstraete, R. Schneider, Ö. Legeza, Tensor product methods and entanglement optimization for ab initio quantum chemistry. Int. J. Quantum Chem. 115, 1342-1391 (2015).

66. G. Barcza, Ö. Legeza, K. H. Marti, M. Reiher, Quantum-information analysis of electronic states of different molecular structures. Phys. Rev. A - At. Mol. Opt. Phys. 83, 012508 (2011).

67. L. V. J. Brabec, J. Brandejs, K. Kowalski, S. Xantheas, O. Legeza, (In Press). J Comp. Chem (2020).

68. S. Szalay, G. Barcza, T. Szilvási, L. Veis, Ö. Legeza, The correlation theory of the chemical bond. Sci. Rep. 7 (2017).

69. D. Schweinfurth, M. Zalibera, M. Kathan, C. Shen, M. Mazzolini, N. Trapp, J. Crassous, G. Gescheidt, F. Diederich, Helicene Quinones: Redox-Triggered Chiroptical Switching and Chiral Recognition of the Semiquinone Radical Anion Lithium Salt by Electron Nuclear Double Resonance Spectroscopy. J. Am. Chem. Soc. 136, 13045-13052 (2014).

70. N. Konstandaras, M. H. Dunn, E. T. Luis, M. L. Cole, J. B. Harper, The p: K a values of Naryl imidazolinium salts, their higher homologues, and formamidinium salts in dimethyl sulfoxide. Org. Biomol. Chem. (2020).

\section{Acknowledgements}

This work has been supported by Praemium Academie of the Academy of Science of the Czech Republic, GACR 18-09914S (P.J., D.N., A.M., B.M., B.T.), GACR 18-18940Y (L.V.) and GACR 18-17823S (M.K.). A. M. and B.M. acknowledges the support from the Internal Student Grant Agency of the Palacký University in Olomouc, Czech Republic IGA_PrF_2020_022 and IGA_PrF_2020_034. The work of the part of the research project RVO of the IOCB of the CAS (61388963).

\section{Contributions}

P.J., and M.K. conceived and designed the experiments. B.T., M.K., D.N., L.V. and P.J. supervised the project and led the collaboration efforts. B.M., B.T. carried out the experiments and obtained the data. M. K. and T.C. synthesized the precursors. The experimental data were analyzed by B.M., P. J. and B.T., and discussed by all the authors. J.M., D.N., A.M., P.M., M.M., L.V. J.B. and P.J. performed the theoretical calculations and data analysis. The manuscript was written by P.J., M.K., D.N., B.T. and L. V. with contribution from all the authors. 
These authors contributed equally: Benjamin Mallada, Bruno de la Torre.

\section{Competing interests}

The authors declare no competing interests.

Data availability

The data that support the findings of this study are available from the corresponding authors upon reasonable request.

\section{Supplementary Materials:}

Materials and Methods

10 Figures S1-S13

Tables S1

Supplementary Information

References (52-70) 
A

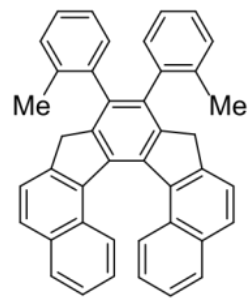

Precursor

$\mathrm{T}>525 \mathrm{~K} \downarrow \mathrm{Au}(111)$

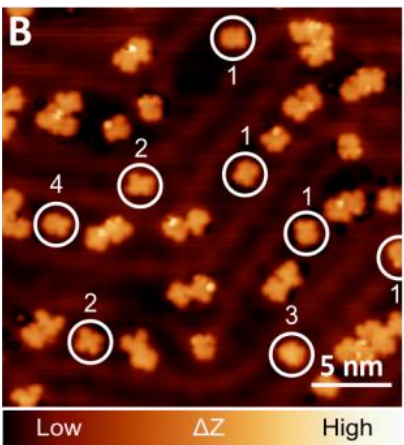

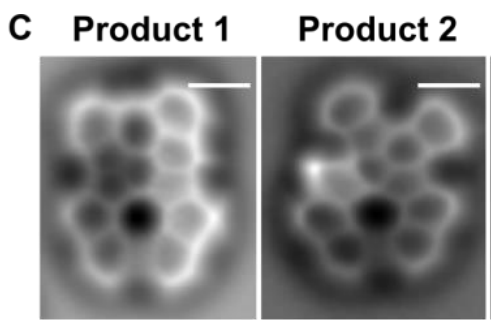

D $\quad 32 \%$

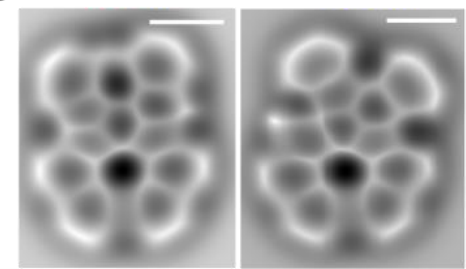

E

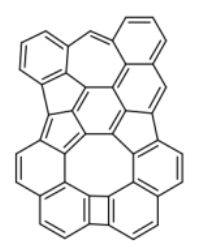

$\Delta \mathrm{E}=0.0 \mathrm{eV}$

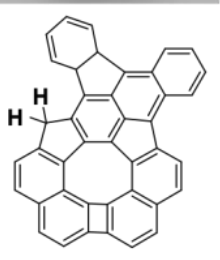

$\Delta \mathrm{E}=5.0 \mathrm{eV}$

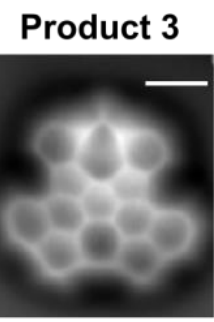

$22 \%$
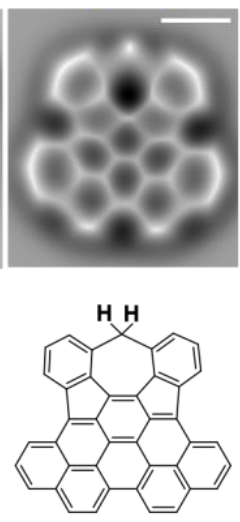

$\Delta \mathrm{E}=2.2 \mathrm{eV}$
Product 4

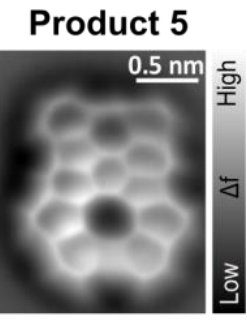

$15 \%$
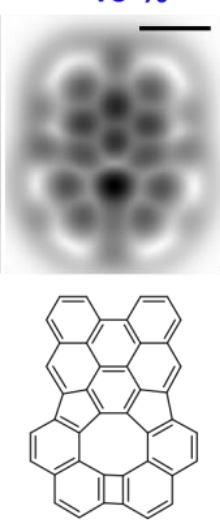

$\Delta \mathrm{E}=-2.2 \mathrm{eV}$
$6 \%$
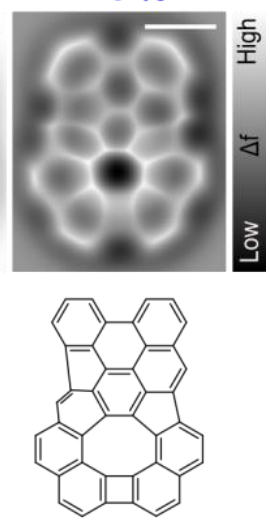

$\Delta \mathrm{E}=-0.9 \mathrm{eV}$

Fig. 1. On-surface synthesis of non-benzenoid molecules including four-eight membered rings; (A) chemical structure of the precursor molecule, which deposited on $\mathrm{Au}(111)$ surface under UHV conditions undergoes chemical transformation upon thermal annealing $>525$ Kelvin. (B) representative constant current STM image of the sample after annealing up to $575 \mathrm{~K}$ shows the presence of various planar and non-planar molecular products. (C) high-resolution AFM images acquired with CO-tip of five planar products found on the surface after annealing; (D) simulated nc-AFM images with CO-tip for five planar products using optimized molecular structure obtained from total energy DFT calculations; (E) chemical structure of the products and corresponding difference of the total energies of molecules on $\mathrm{Au}(111)$ surface with respect to P1 obtained from DFT calculations (for details see SOM). 
A<smiles>COc1ccccc1-c1c2c(c(-c3ccccc3C)c3c1Cc1ccc4ccccc4c1-3)-c1c(ccc3ccccc13)C2</smiles>

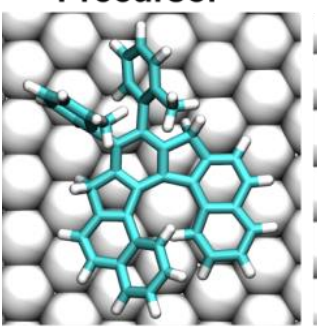

c

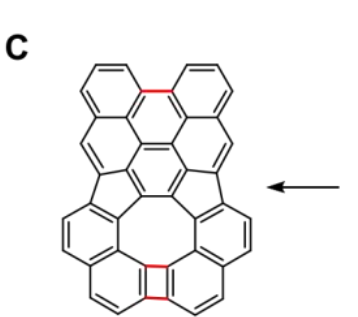

Product 4

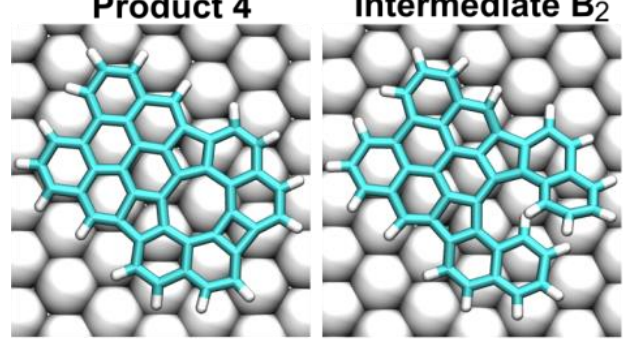

B

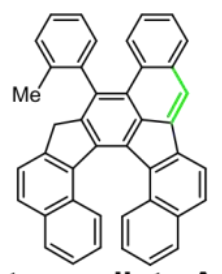
Intermediate $\mathbf{A}_{1}$
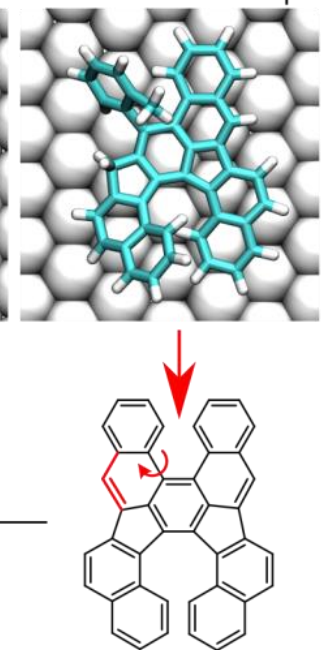
Intermediate $\mathrm{B}_{2}$

D
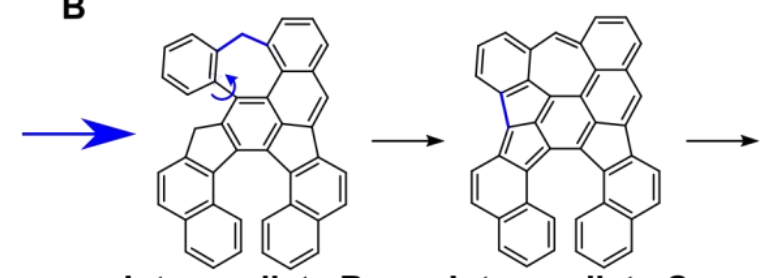

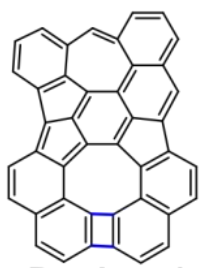

Product 1

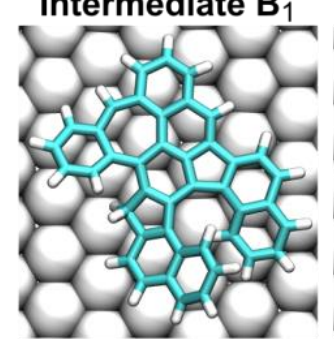

Intermediate $\mathbf{C}_{1}$
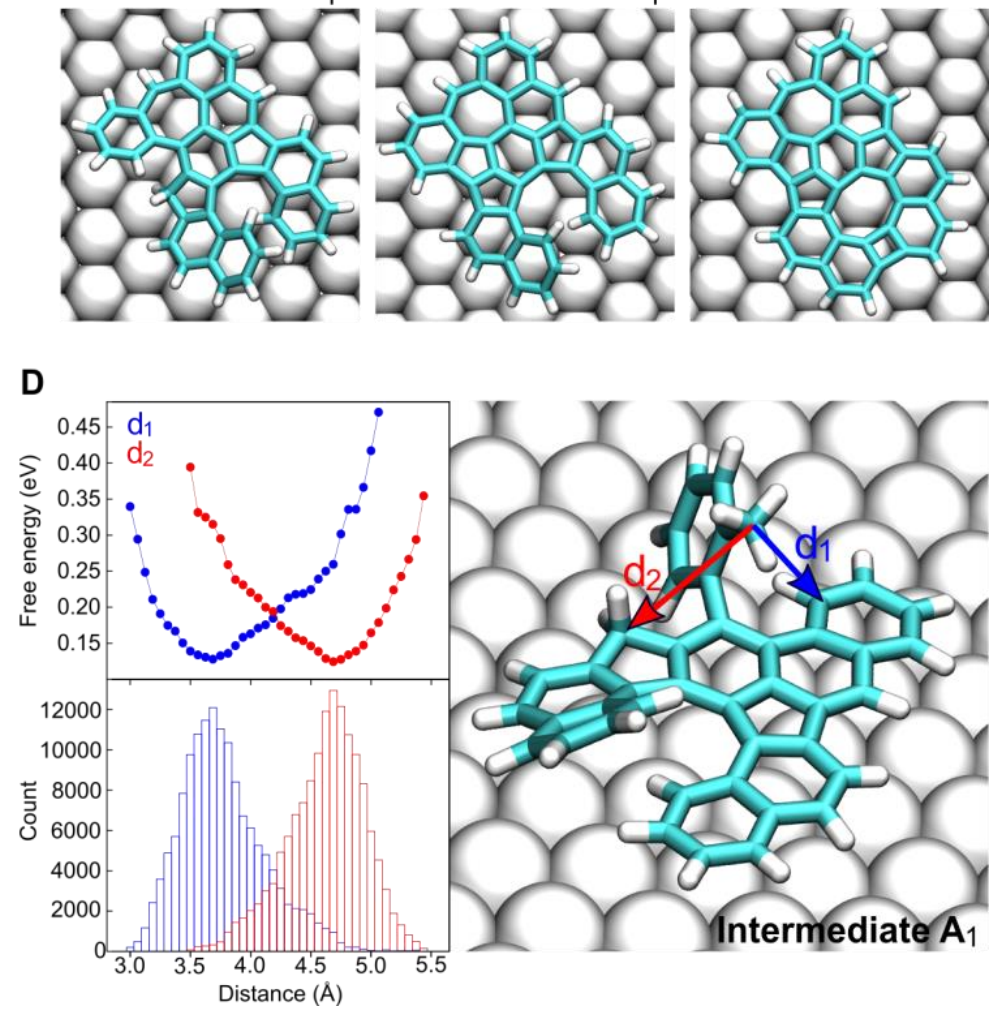

Fig. 2. Course of chemical reaction from precursor towards P1 and P4 products. (A) chemical transformation of the precursor to intermediate A1. Subsequent steps of chemical transformation towards (B) the product P1 and (C) P4. (D) Histogram showing time-distribution of d1 and d2 distances along the reaction coordinates for the reaction steps A1->B1 and A1->B2, respectively calculated by QMMM method at $600 \mathrm{~K}$; and corresponding free energy $\mathrm{F}$ profile derived from the probability $\mathrm{p}$, where $\mathrm{p}=\mathrm{e}^{\wedge}\left(-\Delta \mathrm{F} / \mathrm{k} \_\mathrm{B} \mathrm{T}\right)$ with the probability $\mathrm{p}$ calculated for each interval of the histogram as $\mathrm{p}=($ counts of interval $) /($ total counts). More feasible reaction towards $\mathrm{d} 1$ explains the reaction mechanism enabling to formation of energetically less stable products $\mathrm{P} 1$ instead of $\mathrm{P} 4$. 

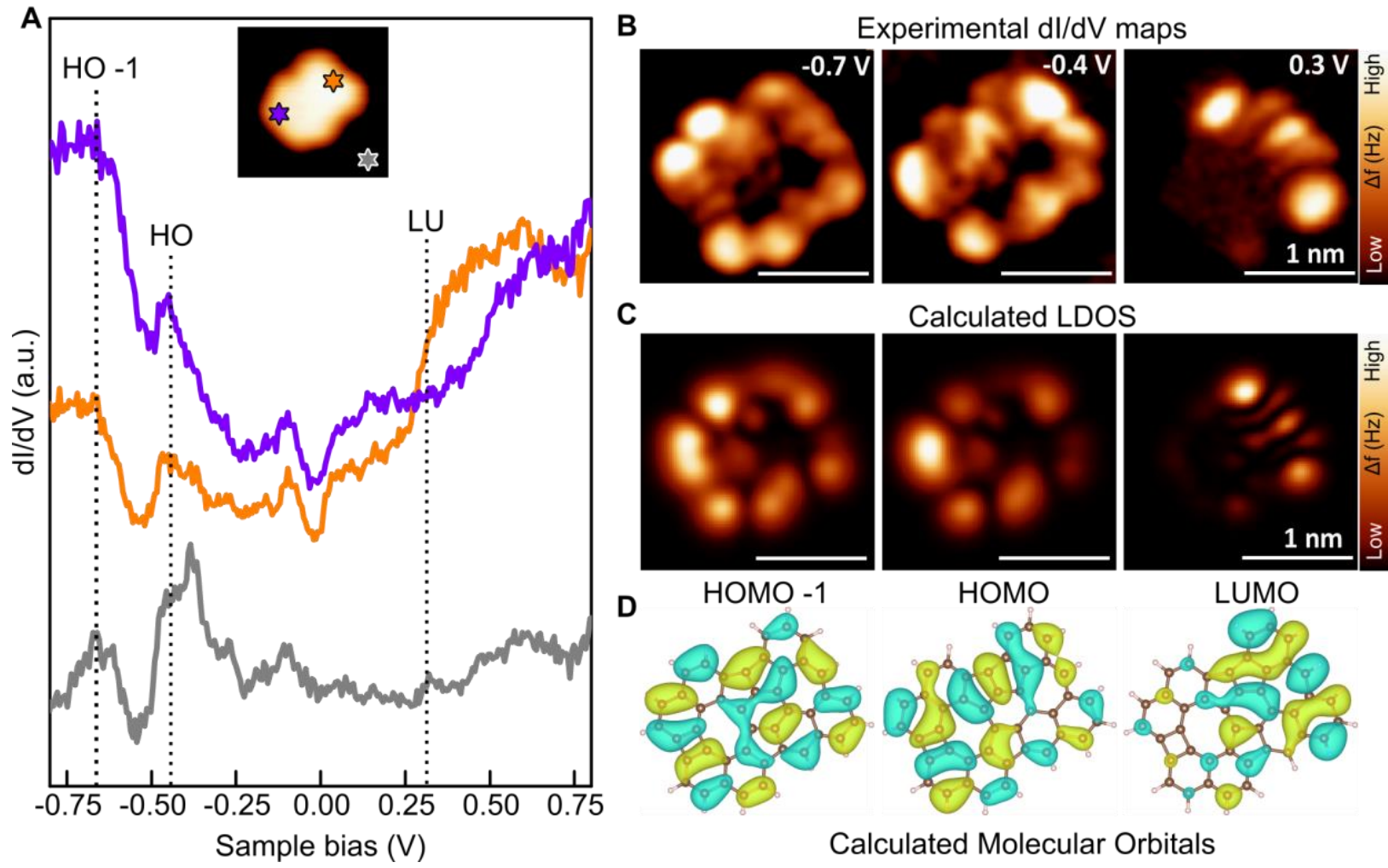

C

Calculated LDOS
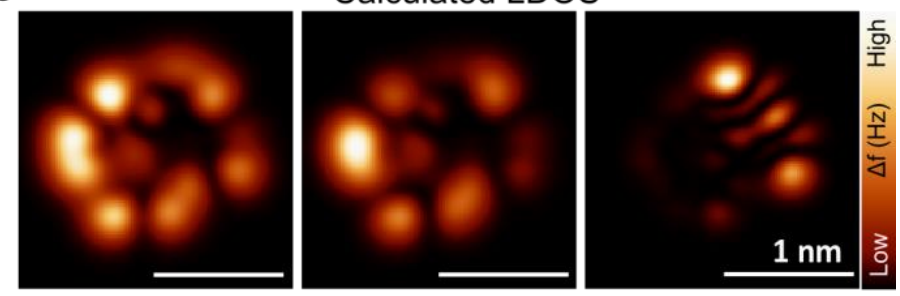

D
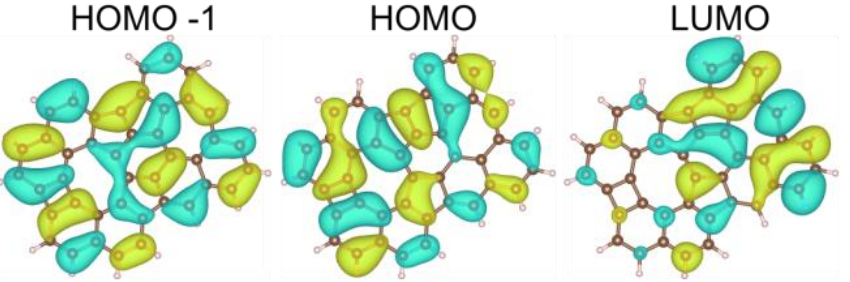

Calculated Molecular Orbitals

Fig. 3. Electronic structure of the product P1. (A) Experimental dI/dV spectra acquired on different sites of $\mathrm{P} 1$ product (see inset); (B) experimental dI/dV maps acquired at three different bias voltages $(-0.7,-0.4$ and $0.3 \mathrm{~V}) ;(\mathbf{C})$ calculated $\mathrm{dI} / \mathrm{dV}$ maps for canonical DFT orbitals of HOMO-1, HOMO and LUMO shown in (D). 

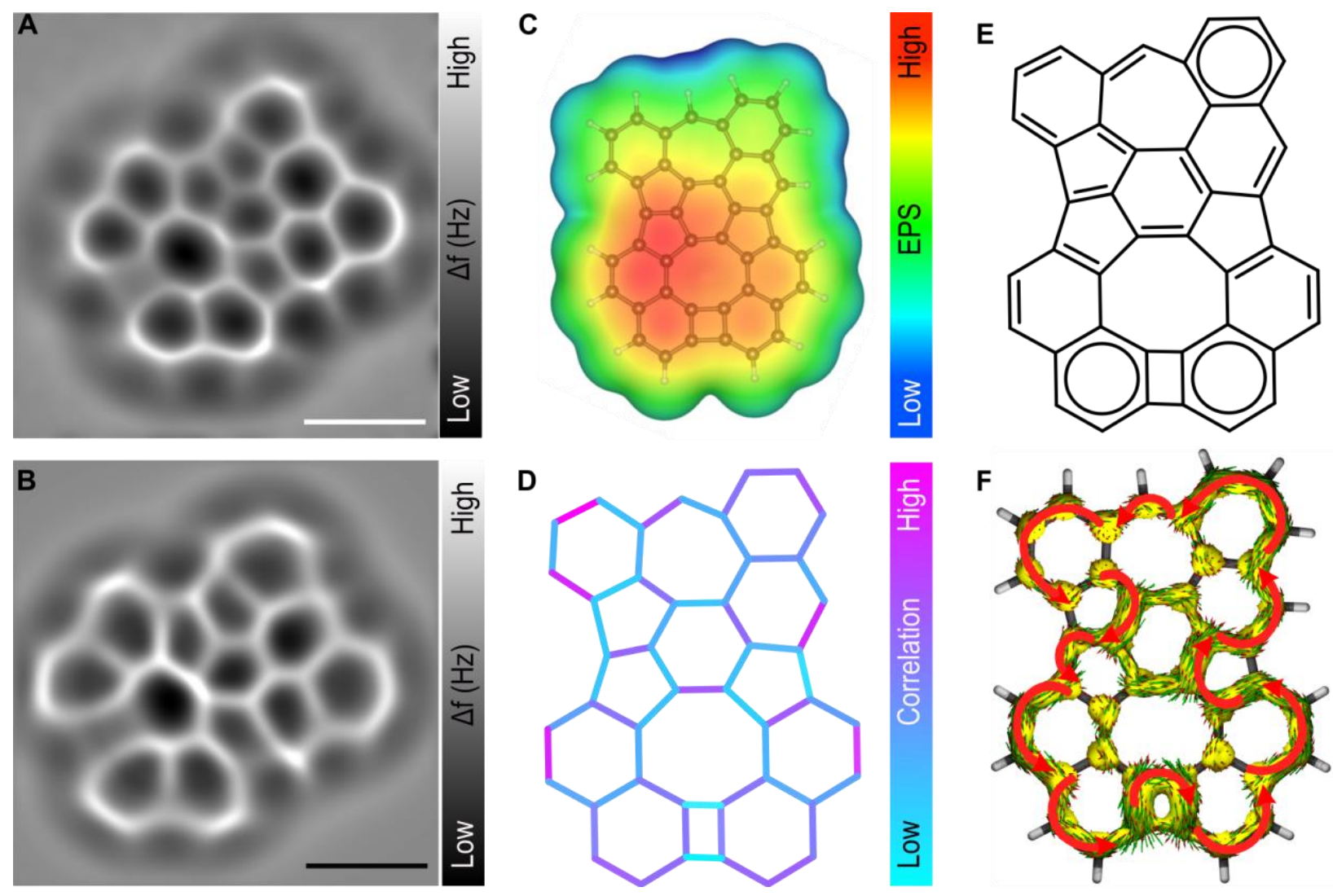

Fig. 4. Analysis of the structure and aromaticity of the product P1. Experimental (A) and simulated (B) high-resolution nc-AFM images of the product P1 obtained with CO-tip (both with adopted Laplace filter); (C) DFT calculated electrostatic potential map of P1; (D) plot of mutual information of individual bonds obtained from DMRG calculations of the product P1; (E) corresponding resonance structure of P1 derived from the mutual information DMRG; (F) plot of calculated ACID of P1 product (red darts show orientation of peripheral ring current). 